\title{
Stronger Lie derivations on MA-semirings
}

\author{
Yaqoub Ahmed ${ }^{1}$. Wieslaw A. Dudek ${ }^{2}$
}

Received: 16 February 2019 / Accepted: 19 January 2020 / Published online: 27 January 2020

(c) The Author(s) 2020

\begin{abstract}
On an additively inverse $M A$-semiring $S$ we consider the additive mapping $f: S \rightarrow S$ satisfying the identity $f(x y)=[x, f(y)]$, where $[a, b]$ is a commutator of $a$ and $b$. We investigate the properties of such a mapping and determine the commutativity of $S$ using this mapping.
\end{abstract}

Keywords Additively inverse semirings · MA-semirings · Semiprime semiring · Centralizing mappings

Mathematics Subject Classification 16 Y60 $\cdot 16$ N60

\section{Introduction}

The notion of a semiring was introduced by Vandiver in 1934 [21]. Semirings which provide a common generalization of rings and distributive lattices appear in a natural manner in some applications to the theory of automata, formal languages, optimization theory and other branches of applied mathematics (cf. [8]). Hemirings, as semirings with commutative addition and zero element, have also proved to be an important algebraic tool in theoretical computer science (cf. $[9,10,20])$.

Nowadays such semirings are studied by many authors in various directions regardless of the derivations in rings where other methods are used (cf. $[2,15,18]$ ). One of the main directions of such studies is investigation of properties of ideals (cf. [1,7,24]). The second important direction is the study of commutativity. For this purpose are used commutators and derivations (cf. [12,19]).

In this paper, we will describe basic properties of the additive mapping $f: S \rightarrow S$ satisfying the identity $f(x y)=[x, f(y)]$ and we will use this mapping to the characterization of commutativity of certain types of $M A$-semirings.

$凶$ Wieslaw A. Dudek

wieslaw.dudek@pwr.edu.pl

Yaqoub Ahmed

yaqoubahmedkhan@gmail.com

1 Department of Mathematics, G.C. University, Lahore, Pakistan

2 Faculty of Pure and Applied Mathematics, Wroclaw University of Science and Technology, Wroclaw, Poland 


\section{Basic definitions and facts}

By a semiring $(S,+, \cdot)$ we mean a nonempty set $S$ equipped with two binary operations + and $\cdot$ (called addition and multiplication) such that the multiplication is distributive with respect to the addition, $(S,+)$ is a semigroup with neutral element 0 , and $(S, \cdot)$ is a semigroup with zero 0 , i.e., $0 a=a 0=0$ for all $a \in S$. If a semigroup $(S, \cdot)$ is commutative, the we say that a semiring $S$ is commutative.

A semiring $S$ is an additively inverse, if for every $a \in S$ there exists a uniquely determined element $a^{\prime} \in S$ such that

$$
a+a^{\prime}+a=a \text { and } a^{\prime}+a+a^{\prime}=a^{\prime} .
$$

Then, according to [14], for all $a, b \in S$ we have

$$
(a b)^{\prime}=a^{\prime} b=a b^{\prime}, \quad(a+b)^{\prime}=b^{\prime}+a^{\prime}, \quad a^{\prime} b^{\prime}=a b, \quad\left(a^{\prime}\right)^{\prime}=a, \quad 0^{\prime}=0 .
$$

Also the following implication is valid

$$
a+b=0 \text { implies } b=a^{\prime} \text { and } a+a^{\prime}=0 .
$$

An additively inverse semiring $S$ with commutative addition satisfying for all $a, b \in S$ the Bandelt and Petrich condition $A_{2}$ (cf. [4]):

$$
\left(a+a^{\prime}\right) b=b\left(a+a^{\prime}\right),
$$

is called an MA-semiring (cf. [13]).

An important role in investigations of such semirings plays the commutator $[x, y]=$ $x y+y^{\prime} x$ and derivations defined as additive mappings $d: S \rightarrow S$ (i.e., endomorphisms of an additive semigroup of $S$ ) such that $d(x y)=d(x) y+x d(y)$ for all $x, y \in S$. If the last identity is valid only for $y=x$, then $d$ is called the Jordan derivation (cf. [11]). The Lie derivation on $S$ is an additive mapping $L: S \longrightarrow S$ satisfying the identity $L([x, y])=[L(x), y]+[x, L(y)]$. Lie derivations are used to the description of algebras of different types (cf. $[3,17,19,25]$ ) and have an important application in differential geometry (cf. [23]).

Let $A$ be a nonempty subset of $S$. An additive mapping is called centralizing on $A$ if [[ $f(x), x], y]=0$ for all $x \in A$ and $y \in S$. In a special case where $[f(x), x]=0$ (or equivalently, $[x, f(x)]=0$ ) for all $x \in A$, the mapping $f$ is said to be commuting on $A$ (cf. [22]), or skew-commuting on $A$ if $f(x) x+x f(x)=0$ for all $x \in A$ (cf. [5] or [6]).

Let's also remind that a semiring $S$ is semiprime if $x S x=0$ implies $x=0$, and prime if $x S y=0$ implies $x=0$ or $y=0$. A semiring $S$ is 2-torsion free if $2 x=0$ is possible only for $x=0$.

We end this section with three simple lemmas that will be useful later.

Lemma 2.1 If $f: S \rightarrow S$ is an additive mapping on an additively inverse semiring $S$, then $f\left(x+x^{\prime}\right) \in Z(S)$ for any $x+x^{\prime} \in Z(S)$, where $Z(S)$ is the center of $S$.

\section{Lemma 2.2 In any $M A$-semiring}

(1) $[x, y]=x y+(y x)^{\prime}=x y+y x^{\prime}$,

(2) $[x, y]^{\prime}=\left[x, y^{\prime}\right]=\left[x^{\prime}, y\right]=[y, x]$,

(3) $\left[x^{\prime}, y^{\prime}\right]=[x, y]$,

(4) $[x, y]=0$ implies $x y=y x$. 
Lemma 2.3 In any MA-semiring the following identities

$$
\begin{aligned}
& {[x y, z]=x[y, z]+[x, z] y \text { and }[x, y z]=y[x, z]+[x, y] z,} \\
& {[z, z]\left(x^{2}+\left(x^{2}\right)^{\prime}\right)=[x, z] x\left(x+x^{\prime}\right)}
\end{aligned}
$$

are valid.

Proof The Jacobi identities (5) are proved in [13]. The proof of (6) is straightforward.

\section{Stronger Lie derivations}

Throughout this paper, $S$ denotes an $M A$-semiring with zero 0 .

Consider the additive mapping $f: S \rightarrow S$ satisfying the identity

$$
f(x y)=[x, f(y)] .
$$

Then, as it is not difficult to see,

$$
f([x, y])=f(x y)+f\left(y^{\prime} x\right)=[f(x), y]+[x, f(y)] .
$$

Hence such mapping is a Lie derivation on an $M A$-semiring $S$ (in an inverse semiring too). Since the converse statement is not true in general, we will call $f$ the stronger Lie derivation (sl-derivation, in short).

Example 3.1 Using the above two lemmas it it is not difficult to see that the mapping $f(x)=$ $[a, x]$ defined on a non-commutative $M A$-semiring $S$ is an example of a Lie derivation which is not a $s l$-derivation. It is a $s l$-derivation in a commutative $M A$-semiring.

Example 3.2 The real vector space $\mathbb{R}^{2}$ with the multiplication of vectors defined by $(a, b)$. $(c, d)=(a c, a d)$ is an $M A$-semiring. The mapping $f(a, b)=(0, a+b)$ is a $s l$-derivation on this $M A$-semiring. This $M A$-semiring is isomorphic to an $M A$-semiring $\mathcal{M}_{2}(S)$ of matrices $\left[\begin{array}{ll}x & y \\ 0 & 0\end{array}\right]$ over an $M A$-semiring $S$ described in [13]. The mapping $f:\left[\begin{array}{ll}x & y \\ 0 & 0\end{array}\right] \mapsto\left[\begin{array}{cc}0 & x+y \\ 0 & 0\end{array}\right]$ is a $s$-derivation on $\mathcal{M}_{2}(S)$.

Example 3.3 The real vector space $\mathbb{R}^{3}$ with the multiplication of vectors defined by $(a, b, c) \cdot(x, y, z)=(0, a z, 0)$ is an $M A$-semiring. The mapping $f(a, b, c)=(0, b, 0)$ is a $s l$-derivation on this $M A$-semiring.

Let's we start with the following simple observations.

Lemma 3.4 Let $f$ be a sl-derivation on an MA-semiring $S$. Then

(1) $f\left(x^{\prime} y\right)=f\left(x y^{\prime}\right)=f(x y)^{\prime}$ for all $x, y \in S$.

(2) If $f\left(x^{2}\right)=0$ for all $x \in S$, then $f$ is commuting.

(3) If $S$ is with unity, then $f(x)=f(x)+f(x)^{\prime}$ for all $x \in S$.

(4) If $S$ is semiprime and $f(x) f(y)=0$ for all $x, y \in S$, then $f=0$.

(5) If $f$ is commuting, then $f\left(x^{n}\right)=0$ for all $n \geq 2$.

(6) If $f(y a)=0$ for all $y \in S$, then $f(a) \in Z(S)$.

Proposition 3.5 If a sl-derivation on a semiprime $M A$-semiring $S$ satisfies the identity $f(a) x+x^{\prime} f(b)=0$, where $a, b \in S$ are fixed, $f(a)=f(b) \in Z(S)$ and $f(y b)=0$ for all $y \in S$. 
Proof By (3), from $f(a) x+x^{\prime} f(b)=0$ it follows $f(a) x=x f(b)$. Therefore, according to the assumption, for all $x, y \in S$ we have

$$
0=f(a) x y+(x y)^{\prime} f(b)=x f(b) y+x^{\prime} y f(b)=x[f(b), y] .
$$

Thus $0=[f(b), y] x[f(b), y]$, which, by semiprimeness and Lemma 2.2, implies $[f(b), y]=0=[f(b), y]^{\prime}=[y, f(b)]=f(y b)$. This, by Lemma 2.2(4), gives $f(b) \in Z(S)$. Therefore, $0=f(a) x+x^{\prime} f(b)=\left(f(a)+f(b)^{\prime}\right) x$, and consequently, $\left(f(a)+f^{\prime}(b)\right) x\left(f(a)+f^{\prime}(b)=0\right.$. Whence, by semiprimeness, we get $f(a)+f(b)^{\prime}=0$. So, $f(a)=f(b)$.

Theorem 3.6 If a sl-derivation $f$ defined on an MA-semiring $S$ satisfies the identity $2 f(x) x=0$, then it is a Jordan derivation. Moreover, if $S$ is 2-torsion free, then $f$ is a derivation.

Proof By (3), from $2 f(x) x=0$ it follows $f(x) x=f(x)^{\prime} x=f(x) x^{\prime}$. Hence $f\left(x^{2}\right)=$ $[x, f(x)]=x f(x)+f(x)^{\prime} x=x f(x)+f(x) x$. So, $f$ is a Jordan derivation.

If $S$ is 2-torsion free, then $2 f(x) x=0$ implies $f(x) x=0$. Thus $f(x)^{\prime} x=0$, and consequently $f\left(x^{2}\right)=x f(x)$. By adding $x^{\prime} f(x)$ to the last equation and using (4), we get

$$
f\left(x^{2}\right)+x^{\prime} f(x)=x f(x)+x^{\prime} f(x)=\left(x+x^{\prime}\right) f(x)=f(x)\left(x+x^{\prime}\right)=0 .
$$

Hence

$$
f\left(x^{2}\right)+x^{\prime} f(x)=0 .
$$

By linearlization of (7) we get $f\left((x+y)^{2}\right)+(x+y)^{\prime} f(x+y)=0$, whence after reduction, we obtain

$$
f(x y+y x)+x^{\prime} f(y)+y^{\prime} f(x)=0 .
$$

Therefore

$$
\begin{aligned}
0 & =f(x y)+f(y x)+x^{\prime} f(y)+y^{\prime} f(x) \\
& =[x, f(y)]+[y, f(x)]+x^{\prime} f(y)+y^{\prime} f(x) \\
& =\left(x+x^{\prime}\right) f(y)+f(y)^{\prime} x+\left(y+y^{\prime}\right) f(x)+f(x)^{\prime} y \\
& \stackrel{(4)}{=} f(y)\left(x+x^{\prime}+x^{\prime}\right)+f(x)\left(y+y^{\prime}+y^{\prime}\right) \\
& =f(y) x^{\prime}+f(x) y^{\prime},
\end{aligned}
$$

which means that

$$
f(y) x+f(x) y=0 .
$$

Consequently,

$$
\begin{aligned}
f(x y)+f(x) y^{\prime}+x^{\prime} f(y) & \stackrel{(2)}{=} x f(y)+f(y) x^{\prime}+f(x) y^{\prime}+x^{\prime} f(y) \\
& =\left(x+x^{\prime}\right) f(y)+f(y) x^{\prime}+f(x) y^{\prime} \\
& \stackrel{(4)}{=} f(y)\left(x+x^{\prime}+x^{\prime}\right)+f(x) y^{\prime} \\
& =f(y) x^{\prime}+f(x) y^{\prime} \stackrel{(8)}{=} 0,
\end{aligned}
$$

which, by (2) and (3), gives $f(x y)=f(x) y+x f(y)$. So, $f$ is a derivation on $S$.

Proposition 3.7 If a sl-derivation $f$ on an MA-semiring $S$ satisfies the identity $f\left(x^{2}\right)+$ $2 x^{\prime} f(x)=0$, then it is skew-commuting. 
Proof Indeed, by (2), we have $0=f\left(x^{2}\right)+2 x^{\prime} f(x)=x^{\prime} f(x)+\left(x f(x)^{\prime}+x f(x)+f(x)^{\prime} x\right)=$ $x^{\prime} f(x)+f(x)^{\prime} x$, which implies $x f(x)+f(x) x=0$. So, $f$ is skew-commuting.

Comparing Proposition 3.7 with Theorem 3.2 in [16] we obtain

Corollary 3.8 In a 2-torsion free semisimple MA-semiring $S$ a sl-derivation $f$ satisfies the identity $f\left(x^{2}\right)+2 x^{\prime} f(x)=0$ if and only if $f(x)=0$ for all $x \in S$.

Proposition 3.9 If a sl-derivation $f$ on an MA-semiring $S$ satisfies the identity $f\left(x^{2}\right)+$ $2 f(x) x^{\prime}=0$, then also

$$
\begin{aligned}
2 f\left(x^{2}\right) & =x f(x)+f(x) x, \\
x f(x) & =3 f(x) x
\end{aligned}
$$

for all $x \in S$.

Proof Indeed, $2 f\left(x^{2}\right)=[x, f(x)]+f\left(x^{2}\right)=x f(x)+\left(f(x)^{\prime} x+2 f(x) x\right)=x f(x)+f(x) x$. This proves (9).

Also $0=f\left(x^{2}\right)+2 f(x) x^{\prime}=x f(x)+3 f(x) x^{\prime}$. This, by (3), implies (10).

Theorem 3.10 If a sl-derivation fdefined on an MA-semiring $S$ satisfies the identity $2 f(x) x=0$, then it also satisfies the identities:

$$
\begin{aligned}
2 f(x y+y x) & =2 x f(y)+2 y f(x), \\
f\left(x y+x^{\prime} y\right) & =2 f(x) y+2 f(y) x, \\
f(x)(x y+y x) & =x f(x) y+y f(x) x .
\end{aligned}
$$

Proof (11) From $2 f(x) x=0$ it follows $f(x) x=f(x) x^{\prime}$ and $f(x) x+f(x) x^{\prime}=0$, which together with (4) gives

$$
f(x)\left(x+x^{\prime}\right)=0=\left(x+x^{\prime}\right) f(x) .
$$

Consider the equation $f\left((x+y)^{2}\right)=f\left(x^{2}\right)+f\left(y^{2}\right)+f(x y+y x)$. Since, by Theorem 3.6, $f$ is a Jordan derivation, the last equation can be rewritten in the form

$(x+y) f(x+y)+(x+y) f(x+y)=x f(x)+f(x) x+y f(y)+f(y) y+f(x y+y x)$.

By adding $x^{\prime} f(x)+f(x) x^{\prime}+y^{\prime} f(y)+f(y) y^{\prime}$ to both sides of the last equation and using (14) we get

$$
f(x y+y x)=x f(y)+y f(x)+f(x) y+f(y) x .
$$

Thus, $f(x y+y x)=[x, f(y)]+[y, f(x)]$, or in the other words

$$
f(x y+y x)=x f(y)+f(y) x^{\prime}+y f(x)+f(x) y^{\prime} .
$$

Adding (16) to (15) we obtain (11).

Further we have

$$
\begin{aligned}
& f\left(x y+x^{\prime} y\right)=f\left(\left(x+x^{\prime}+x\right) y+x y^{\prime}\right)=f\left(\left(x+x^{\prime}\right) y+x\left(y+y^{\prime}\right)\right) \\
& \quad \stackrel{(4)}{=} f\left(x y+y x+x^{\prime} y+y^{\prime} x\right)=f(x y+y x)+f(x y+y x)^{\prime} \\
& \quad \stackrel{(15),(16)}{=} x f(y)+y f(x)+f(x) y+f(y) x+x^{\prime} f(y)+f(y) x+y^{\prime} f(x)+f(x) y \\
& \quad=\left(x+x^{\prime}\right) f(y)+2 f(y) x+\left(y+y^{\prime}\right) f(x)+2 f(x) y \\
& \quad \stackrel{(4)}{=} f(y)\left(x+x^{\prime}\right)+2 f(y) x+f(x)\left(y+y^{\prime}\right)+2 f(x) y \\
& \quad=f(y)\left(x+x^{\prime}+2 x\right)+f(x)\left(y+y^{\prime}+2 y\right) \\
& \quad=2 f(y) x+2 f(x) y .
\end{aligned}
$$


This proves (12).

Now from $f(x y x)=f(x(y x))=[x, f(y x)]=x[y, f(x)]+[y, f(x)] x^{\prime}$, we calculate

$$
f(x y x)=x y f(x)+x f(x) y^{\prime}+y f(x) x^{\prime}+f(x) y x .
$$

Analogously

$$
f(x y x)=f((x y) x)=[x y, f(x)]=x y f(x)+f(x) x y^{\prime} .
$$

Using these two identities we obtain

$$
\begin{aligned}
& f\left(\left(x+x^{\prime}\right) y x\right)=f(x y x)+f(x y x)^{\prime} \\
& \quad=x y f(x)+x f(x) y^{\prime}+y f(x) x^{\prime}+f(x) y x+x^{\prime} y f(x)+f(x) x y \\
& \quad=\left(x+x^{\prime}\right) y f(x)+x f(x) y^{\prime}+y f(x) x^{\prime}+f(x) y x+f(x) x y \\
& \stackrel{(4),(14)}{=} x f(x) y^{\prime}+y f(x) x^{\prime}+f(x) y x+f(x) x y \\
& =(x f(x) y+y f(x) x)^{\prime}+f(x)(y x+x y) .
\end{aligned}
$$

But on the other hand, in view of (4) and (14), we also have

$$
f\left(\left(x+x^{\prime}\right) y x\right)=\left[\left(x+x^{\prime}\right) y, f(x)\right]=0 .
$$

Thus $(x f(x) y+y f(x) x)^{\prime}+f(x) y x+f(x) x y=0$, which by (3) implies (13). This completes the proof.

\section{Commutativity of $M A$-semirings}

In this section we characterize the commutativity of $M A$-semirings by the properties of some their ideals and a $s l$-derivation.

Let's we start with the following three simple lemmas.

Lemma 4.1 Let $S$ be a semiprime MA-semiring. Then any element a $\in S$ satisfying the identity $a[a, x]=0$ is in the center of $S$.

Proof By hypothesis, $a[a, x y]=0$ for all $x, y \in S$. Hence, applying Lemma 2.3, we obtain $a x[a, y]+a[a, x] y=0$. Thus $a x[a, y]=0$. In particular, for $x=y x$ we have $a y x[a, y]=0$. Multiplying the previous identity by $y^{\prime}$ and adding to the last we obtain $[a, y] x[a, y]=0$. So, $[a, y]=0$ because $S$ is semiprime. Lemma 2.2 completes the proof.

Lemma 4.2 Let $U$ be a non-zero right ideal of a semiprime MA-semiring $S$ and $a \in U$. If $[a, x]=0$ for all $x \in U$, then $a \in Z(S)$.

Proof Indeed, ar $\in U$ for each $r \in S$. Thus, according to the assumption, $0=[a, a r]=$ $a[a, r]$. So, by Lemma 4.1, $a \in Z(S)$.

Lemma 4.3 The center of a semiprime semiring does not contain any non-zero nilpotent elements.

Proof Let $a \in Z(S)$ be a nilpotent element of index $n$. If $n=2$, then $0=a^{2} s=a s a$ for each $s \in S$. This implies $a=0$. If $n>2$, then $a^{m}=0$ for each $m \geq n$. Thus $0=a^{2 n-2}=a^{2 n-2} s=a^{n-1} s a^{n-1}$ for each $s \in S$. Hence $a^{n-1}=0$. This is possible only for $a=0$. 
Theorem 4.4 Let $f$ be a non-zero sl-derivation on a prime $M A$-semiring $S$. If $U$ is a non-zero right ideal of $S$ such that $f(U)=0$, then $f(S) \subseteq Z(S)$ and $f$ is commuting on $S$.

Proof For all $a \in U$ and $s \in S$ we have $0=f(a s)=[a, f(s)]$. Thus also $f(s)[f(s), a]=0$, whence, by Lemma 4.1, $f(S) \subseteq Z(S)$. Moreover, by Lemma 2.3, for all $s \in S$ we also have $0=f((a s) s)=[a s, f(s)]=a[s, f(s)]+[a, f(s)] s=a[s, f(s)]$. Because $x S \subseteq U$ for each $x \in U$, the last implies $x S[s, f(s)]=0$, whence $[s, f(s)]=0$ for all $s \in S$. So, $f$ is commuting on $S$.

Let's remind that the Lie ideal of a semiring $S$ is defined as an additive subsemigroup $U$ of $S$ such that $[u, s]=u s+s^{\prime} u \in U$ for all $u \in U$ and $s \in S$.

Proposition 4.5 Let $f$ be a non-zero sl-derivation on a prime $M A$-semiring $S$. If $U$ is a non-zero Lie ideal of $S$ such that $f(U)=0$, then $f(S) \subseteq Z(U)$. Moreover, if $U$ is not a ring, then $f$ is centralizing on $S$.

Proof Since $f(u)=0$ for $u \in U$, for all $u \in U$ and $x \in S$ we have $0=f\left(u x+x^{\prime} u\right)=$ $[u, f(x)]+\left[x^{\prime}, f(u)\right]=[u, f(x)]$. Hence, by Lemma 4.2, $f(S) \subseteq Z(S)$.

From $0=[u, f(x)]$, for $u:=u x+x^{\prime} u$, we obtain $0=\left[u x+x^{\prime} u, f(x)\right]=u[x, f(x)]+$ $[x, f(x)] u^{\prime}=[u,[x, f(x)]]=\left[u, f\left(x^{2}\right)\right]$. This for $u:=u r+r^{\prime} u$, where $r \in S$, gives $0=u\left[r, f\left(x^{2}\right)\right]+\left[r, f\left(x^{2}\right)\right] u^{\prime}=\left[u,\left[r, f\left(x^{2}\right)\right]\right]$, whence by Lemma 4.2, $\left[r, f\left(x^{2}\right)\right] \in$ $Z(S)$. Thus $0=\left[u,\left[r, f\left(x^{2}\right)\right]\right]=\left(u+u^{\prime}\right)\left[r, f\left(x^{2}\right)\right]$. So, $u+u^{\prime}=0$ or $\left[r, f\left(x^{2}\right)\right]=$ $[r,[x, f(x)]]=0$. As $U$ is not a ring, must be $[r,[x, f(x)]]=0$. This completes the proof.

Proposition 4.6 Let $f$ be a sl-derivation of an MA-semiring $S$ such that $f(u)+u^{\prime}=0$ for all elements of some subset $U \subseteq S$.

(a) If $U$ is a left ideal of $S$, then

$$
U \subseteq l(S)=\{y \in S: y x=0 \text { for all } x \in S\} .
$$

Moreover, if $S$ is semiprime, then $U=\{0\}$.

(b) If $U$ is a right ideal of $S$, then $u^{2} \in Z(S)$ for all $u \in U$. Moreover, if $S$ is prime, then $U=\{0\}$ or $S$ is a ring.

Proof Let's notice first that $f(u)+u^{\prime}=0$ implies $f(u)=u$ and $u+u^{\prime}=0$.

(a). If $U$ is a left ideal, then for $u \in U$ and $s \in S$ we have $s u \in U$ and $0=f(s u)+s u^{\prime}=$ $[s, f(u)]+s u^{\prime}=[s, u]+s u^{\prime}=u s^{\prime}$, because $u+u^{\prime} \in Z(S)$. Thus $u S=\{0\}$. The rest is obvious.

(b). Let $U$ be a right ideal. Then for all $u \in U$ and $s \in S$ we have $0=f(u(s u))+$ $(u(s u))^{\prime}=u s u+u^{2} s^{\prime}+s^{\prime} u^{2}$ and $0=f(u(u s))+(u(u s))^{\prime}=u^{2} s+u s u^{\prime}+u^{2} s^{\prime}$. Adding up these two identities we obtain $u^{2} s+s^{\prime} u^{2}=0$. So, by (3), $u^{2} \in Z(S)$.

Moreover, in this case also $0=u^{2}\left(s+s^{\prime}\right)=u\left(u s+u s^{\prime}\right)$. But $S$ is a $M A$-semiring, hence $u s+u s^{\prime} \in Z(S)$, and consequently, $0=u S\left(u s+s u^{\prime}\right)$ Thus, if $S$ is prime, $u=0$ or $u\left(s+s^{\prime}\right)=0$ for all $s \in S$. So, $0=u\left(s+s^{\prime}\right) S=u S\left(s+s^{\prime}\right)$ again implies $u=0$ or $s+s^{\prime}=0$. Therefore $U=\{0\}$ or $S$ is a ring.

Proposition 4.7 If a sl-derivation $f$ on a prime $M A$-semiring $S$ is injective on a non-zero left ideal $U$ of $S$, then it is injective on whole $S$.

Proof Indeed, $(\operatorname{ker} f) S U \subseteq(\operatorname{ker} f) U \subseteq(\operatorname{ker} f) \cap U=\{0\}$, because $U$ is a non-zero left ideal and $f$ is injective on $U$. Thus ker $f=\{0\}$. 
Theorem 4.8 If a sl-derivation $f$ on a semiprime $M A$-semiring $S$ is centralizing on an ideal $U$ of $S$, then it is commuting on $U$.

Proof As $f$ is centralizing on $U$, therefore

$$
[[f(x), x], z]=0
$$

for all $x \in U$ and $z \in S$. This, by Lemma 2.2, means that $[f(x), x] \in Z(S)$. Now, replacing in (17) an element $x$ by $x+y$, where $y \in U$, we get

$$
[[f(x), y]+[f(y), x], z]=0 .
$$

From this, for $y=x^{2}$ and $z=f(x)$, we obtain

$$
\begin{aligned}
0 & =\left[\left[f(x), x^{2}\right]+\left[f\left(x^{2}\right), x\right], f(x)\right] \\
& =[x[f(x), x]+[f(x), x] x+[[x, f(x)], x], f(x)] \\
& \stackrel{(17)}{=}[2 x[f(x), x], f(x)]=2(x[[f(x), x], f(x)]+[x, f(x)][f(x), x]) \\
& =2[x, f(x)][f(x), x]=2[f(x), x]^{2} .
\end{aligned}
$$

Consequently, $0=2[f(x), x]^{2}+2[f(x), x]^{2}=4[f(x), x]^{2}=(2[f(x), x])^{2}$. This, by Lemma 4.3, means that

$$
2[f(x), x]=0 .
$$

Replacing in this equation $x$ by $x+y, x, y \in U$, we obtain

$$
2([y, f(x)]+[x, f(y)])=0 .
$$

Now using Lemma 2.3 we have

$$
\begin{aligned}
& {[x y+y x, f(x)]+\left[x^{2}, f(y)\right]=[x y, f(x)]+[y x, f(x)]+x[x, f(y]+[x, f(y)] x} \\
& \quad=x[y, f(x)]+[x, f(x)] y+y[x, f(x)]+[y, f(x)] x+x[x, f(y)]+[x, f(y)] x \\
& \quad \stackrel{(18)}{=} 2 y[x, f(x)]+2 x([y, f(x)]+[x, f(y)]) \stackrel{(19),(20)}{=} 0 .
\end{aligned}
$$

Therefore,

$$
[x y+y x, f(x)]+\left[x^{2}, f(y)\right]=0
$$

for $x, y \in U$.

Since for $x \in U$ elements $[f(x), x],[x, f(x)],[f(x), f(x)]=[f(x), f(x)]^{\prime}$ are in the center of $S$, replacing in the above equation $y$ by $f(x) x$ and using Lemma 2.2 we obtain

$$
\begin{aligned}
0 & =[x f(x) x+f(x) x x, f(x)]+\left[x^{2}, f(f(x) x)\right] \\
& \stackrel{(1)}{=}\left[\left(x f(x)+f^{\prime}(x) x+f(x) x+f(x) x\right) x, f(x)\right]+\left[x^{2},[f(x), f(x)]\right] \\
= & {[([x, f(x)]+2 f(x) x) x, f(x)]+\left[x^{2},[f(x), f(x)]\right] } \\
= & {[[x, f(x)] x, f(x)]+\left[2 f(x) x^{2}, f(x)\right]+x^{2}[f(x), f(x)]+[f(x), f(x)] x^{2} } \\
& \stackrel{(18)}{=}[x, f(x)]^{2}+2 f(x)\left[x^{2}, f(x)\right]+2[f(x), f(x)] x^{2}+[[x, f(x)], f(x)] x \\
& +2[f(x), f(x)] x^{2} \\
& \stackrel{(5)}{=}[x, f(x)]^{2}+2 f(x) x[x, f(x)]+2 f(x)[x, f(x)]+[x, f(x)] f(x) x
\end{aligned}
$$




$$
\begin{aligned}
& \quad+f(x)^{\prime}[x, f(x)] x+2[f(x), f(x)] x^{2}+2[f(x), f(x)]\left(x^{2}\right)^{\prime} \\
& \stackrel{(18)}{=}[x, f(x)]^{2}+[x, f(x)] f(x) x+f(x)^{\prime}[x, f(x)] x \\
& \quad+[f(x), f(x)]\left(x^{2}+x^{2}+\left(x^{2}\right)^{\prime}+\left(x^{2}\right)^{\prime}\right) \\
& \stackrel{(1)}{=}[x, f(x)]^{2}+[x, f(x)] f(x)\left(x+x^{\prime}\right)+[f(x), f(x)]\left(x^{2}+\left(x^{2}\right)^{\prime}\right) \\
& \stackrel{(6)}{=}[x, f(x)]^{2}+2[x, f(x)] f(x)\left(x+x^{\prime}\right) \stackrel{(19)}{=}[x, f(x)]^{2} .
\end{aligned}
$$

Thus $0=[x, f(x)]^{2}=[x, f(x)] S[x, f(x)]$. As $S$ is semiprime, the last implies $[x, f(x)]=0$ and hence $f$ is commuting on $U$.

Corollary 4.9 Any sl-derivation centralizing on whole semiprime MA-semiring is also commuting on this semiring.

By Proposition 4.5 and Corollary 4.9, we get the following result.

Corollary 4.10 Let $f$ be a sl-derivation of a prime $M A$-semiring $S$. If $S$ has a non-zero Lie ideal that is not a ring and $f(x)=0$ for all $x \in U$, then $f$ is commuting on $S$.

Proposition 4.11 Let $U$ be a non-zero right ideal of a prime MA-semiring S. If a sl-derivation $f$ is surjective and $f(u v)=0$ for all $u, v \in U$, then $U$ is a ring or $S$ is commutative.

Proof Since $f$ is surjective for every $z \in S$ there is $s \in S$ such that $z=f(s)$ and $u s \in U$ for all $u \in U$. Thus $0=f(u(u s))=[u,[u, f(s)]]=[u,[u, z]]=\left(u+u^{\prime}\right)[u, z]$. Hence $\left(u+u^{\prime}\right) S[u, z]=0$, which implies $u+u^{\prime}=0$ or $[u, z]=0$ for all $z \in S$ and $u \in U$. In the first case $U$ is a ring; in the second $S$ is commutative by Lemma 4.2.

Theorem 4.12 If a semiprime MA-semiring has a centralizing surjective sl-derivation, then it is commutative.

Proof Let $f$ be a centralizing $s l$-derivation on $S$. By Theorem 4.8, it is commuting on $S$. Therefore $[f(x), x]=0$ holds for all $x \in S$. From this, putting $x=y+z$, we can see that

$$
[f(x), y]+[x, f(y)]^{\prime}=0,
$$

and consequently, by (3),

$$
[f(y), z]=[y, f(z)]
$$

for all $y, z \in S$. Replacing in this identity $y$ by $z x$, we obtain

$$
[f(z x), z]=[z x, f(z)]=z[x, f(z)]+[z, f(z)] x=z[x, f(z)] .
$$

But by definition, $[f(z x), z]=[[z, f(x)], z]$. Therefore, by Lemma 2.2,

$$
\begin{aligned}
& {[f(z x), z]+[f(z x), z]^{\prime}=z[x, f(z)]+[[z, f(x)], z]^{\prime}} \\
& \quad=z[x, f(z)]+[z,[z, f(x)]]=z[x, f(z)]+z[z, f(x)]+[z, f(x)]^{\prime} z \\
& \quad \stackrel{(23)}{=} z[f(x), z]+z[z, f(x)]+[z, f(x)] \stackrel{(1),(4)}{=}[z, f(x)] z^{\prime}=0,
\end{aligned}
$$

because

$$
\begin{aligned}
& {[f(z x), z]+[f(z x), z]^{\prime} \stackrel{(23)}{=}[z x, f(z)]+[z x, f(z)]^{\prime}} \\
& \quad \stackrel{(5)}{=} z[x, f(z)]+[z, f(z)] x+z[x, f(z)]^{\prime}+[z, f(z)]^{\prime} x
\end{aligned}
$$




$$
=z[x, f(z)]+z[x, f(z)]^{\prime} \stackrel{(23)}{=} z\left([f(x), z]+[x, f(z)]^{\prime}\right) \stackrel{(22)}{=} 0 .
$$

So, $0=[z, f(x)] z^{\prime}$, i.e., $0=[z, w] z$ for all $w, z \in S$ since $f$ is surjective. From this, by putting $w=s u$ and using (5), we obtain $0=[z, s] u z$ for all $s, u, z \in S$. Consequently, $0=[z, s] u z s$ and $0=[s, z] u s z=[z, s] u z^{\prime} s$. Thus, $[s, z] S[s, z]=0$, which implies $[s, z]=0$. This by last statement of Lemma 2.2 means that $S$ is a commutative semiring.

Open Access This article is licensed under a Creative Commons Attribution 4.0 International License, which permits use, sharing, adaptation, distribution and reproduction in any medium or format, as long as you give appropriate credit to the original author(s) and the source, provide a link to the Creative Commons licence, and indicate if changes were made. The images or other third party material in this article are included in the article's Creative Commons licence, unless indicated otherwise in a credit line to the material. If material is not included in the article's Creative Commons licence and your intended use is not permitted by statutory regulation or exceeds the permitted use, you will need to obtain permission directly from the copyright holder. To view a copy of this licence, visit http://creativecommons.org/licenses/by/4.0/.

\section{References}

1. Ahsan, J.: Semirings characterized by their fuzzy ideals. J. Fuzzy Math. 6, 181-192 (1998)

2. Albaş, E.: Generalized derivations on ideals of prime rings. Miskolc Math. Notes 14, 3-9 (2013)

3. Bai, Z., Du, S.: The structure of nonlinear Lie derivation on von Neumann algebras. Linear Algebra Appl. 436, 2701-2708 (2012)

4. Bandelt, H.J., Petrich, M.: Subdirect products of rings and distrbutive lattics. Proc. Edin Math. Soc. 25, 155-171 (1982)

5. Bell, H.E., Lucier, J.: On additive maps and commutativity in rings. Result. Math. 36, 1-8 (1999)

6. Brešar, M.: On skew-commuting mappings of rings. Bull. Aust. Math. Soc. 47, 291-296 (1993)

7. Dudek, W.A.: Special types of intuitionistic fuzzy left h-ideals of hemirings. Soft Comput. 12, 359-364 (2008)

8. Glazek, K.: A Guide to Literature on Semirings and their Applications in Mathematics and Information Sciences with Complete Bibliography. Kluwer Academic Publisher, Dodrecht (2002)

9. Golan, J.S.: Semirings and Their Applications. Kluwer Academic Publisher, Dodrecht (1998)

10. Hebisch, U., Weinert, H.J.: Semirings: Algebraic Theory and Applications in the Computer Science. World Scientific, Singapore (1998)

11. Herstein, I.N.: Jordan derivations on prime rings. Proc. Am. Math. Soc. 8, 1104-1110 (1957)

12. Javed, M.A., Aslam, M.: Some commutativity conditions in prime MA-semirings. Ars Comb. 114, 373384 (2014)

13. Javed, M.A., Aslam, M., Hussain, M.: On condition $\left(A_{2}\right)$ of Bandelt and Petrich for inverse semirings. Int. Math. Forum 7, 2903-2914 (2012)

14. Karvellas, P.H.: Inversive semirings. J. Austral. Math. Soc. 18, 277-288 (1974)

15. Koç, E., Gölbaşi, Ö.: Multiplicative generalized derivations on Lie ideals in semiprime rings II. Miskolc Math. Notes. 18, 265-276 (2017)

16. Nadeem, M., Aslam, M.: On the generalization of Brešar theorems. Quasigroups Relat. Syst. 24, 123-128 (2016)

17. Qi, X., Hou, J.: Characterization of Lie derivations on prime rings. Commun. Algebra 39, 3824-3835 (2011)

18. Rehman, N., Arif Raza, M.: On ideals with skew derivations of prime rings. Miskolc Math. Notes. 15, 717-724 (2014)

19. Shafiq, S., Aslam, M.: Centralizers on semiprime MA-semirings. Quasigroups Relat. Syst. 24, 269-276 (2016)

20. Sokolova, A., Woracek, H.: Proper semirings and proper convex functors. Foundations of software science and computation structures. Lect. Notes Comput. Sci. 10803, 331-347 (2018)

21. Vandiver, H.S.: Note on a simple type of algebra in which cancellation law of addition does not hold. Bull. Am. Math. Soc. 40, 914-920 (1934)

22. Vukman, J.: Commuting and centralizing mappings in prime rings. Proc. Am. Math. Soc. 109, 47-52 (1990)

23. Yano, K.: The Theory of Lie Derivatives and Its Applications. North Holland, Amsterdam (1957) 
24. Zhan, J., Dudek, W.A.: Fuzzy h-ideals of hemirings. Inform. Sci. 177, 876-886 (2007)

25. Zhang, X., Khrypchenko, M.: Lie derivations of incidence algebras. Linear Algebra Appl. 513, 69-83 (2017)

Publisher's Note Springer Nature remains neutral with regard to jurisdictional claims in published maps and institutional affiliations. 